\title{
PEOPLE 3000 working group
}

Julie A. Hoggarth', C. Latorre², J. Freeman 3 , E. Robinson 4 , E. Gayo ${ }^{5}$ and D. Bird ${ }^{6,7}$

Understanding what makes some socioecological systems (SES) more resilient to changing disturbance regimes (e.g. the length of fire season caused by abrupt climate change) is integral to explain longterm patterns in the development of human societies over the past 3,000 years. The PAGES PalEOclimate and the PeopLing of Earth (PEOPLE 3000; pastglobalchanges.org/ people3000) working group explores this question by integrating archaeological data, paleoecological data, and dynamic modeling (Fig. 1) to identify regionally comparative case studies across the world.

Humans adapt to natural conditions, including variability in climate systems, over time with each generation inheriting ecological and cultural knowledge. Drawing from niche construction theory, we further argue that humans modify selection pressures in their environments that affect both humans and other species (Odling-Smee et al. 2003). As disturbances may change over time, an SES may become vulnerable when those changes extend outside of the range held within the cultural and ecological memory of a society.

More flexible systems may improve the resilience of SESs over even highly productive but rigid systems. Using radiocarbon datasets as proxies of populations, Freeman et al. (2021) explored whether systems with greater variability in production developed differing population stability patterns than those with more landscape engineering over time. They found that agricultural societies that relied on landscape engineering to intensify production and control variability of production experienced the most stability and the least severe population declines during times of environmental stress.

\section{Scientific goals and activities}

The PEOPLE 3000 working group has three primary goals: (1) to develop low- and highprecision coupled records of paleoclimate, human population, and human institutions over the last 3000 years; (2) compare changes in population, paleoclimate, and institutions from region to region; and (3) identify regionally comparative patterns to explain relationships between variation in ecosystem change, subsistence and social diversity, and the severity of socialecological reorganization. To explore these questions, we are building a global data infrastructure for comparing patterns of human population ecology. To date, in Phase of PEOPLE 3000, this has taken the form of a global radiocarbon database, compiling,

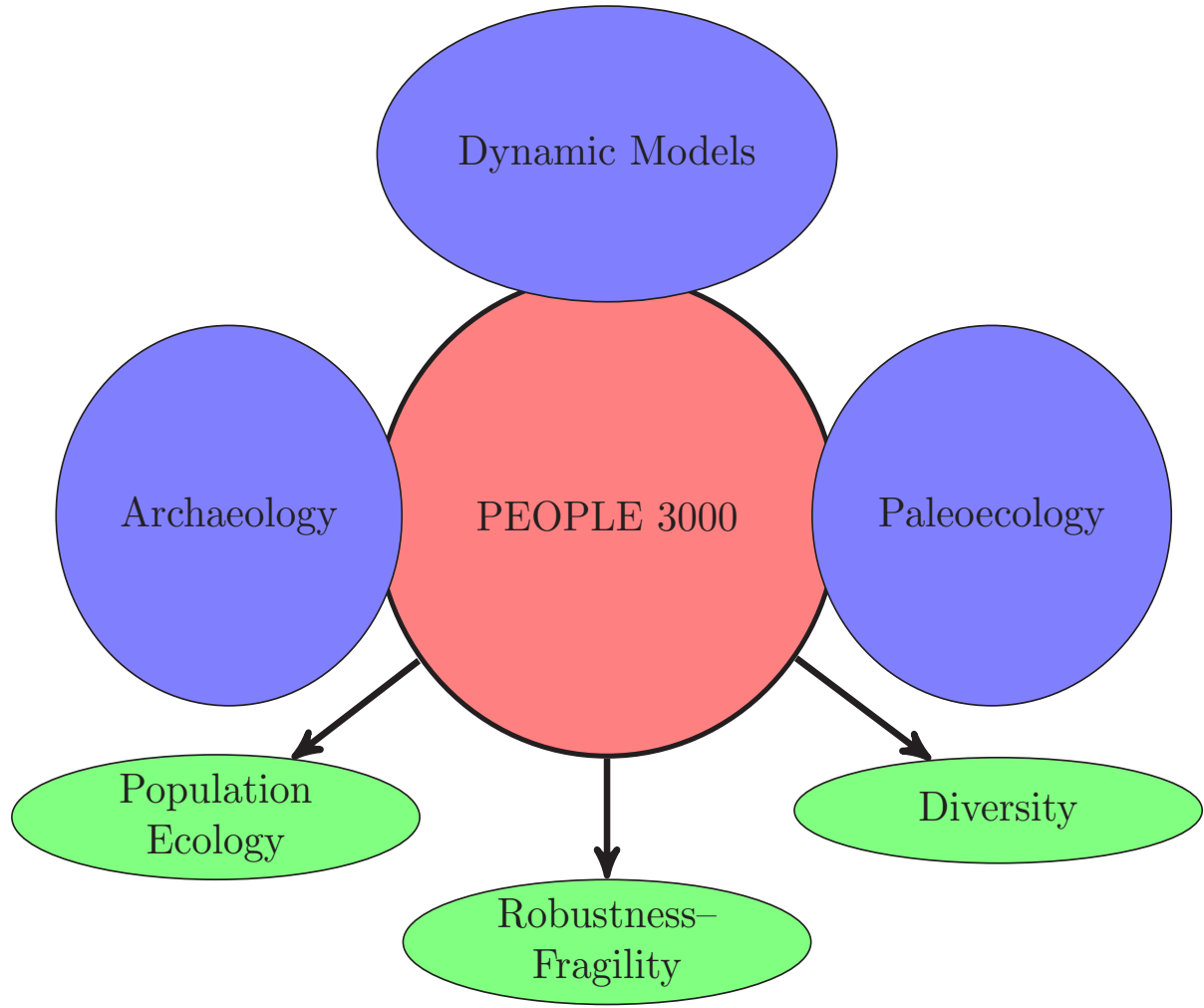

Figure 1: Conceptual diagram of PEOPLE 3000, showing the three areas of archaeology, dynamic models, and paleoecology that are integrated in the project.

and curation of over 150,000 radiocarbon dates from around the world and developing protocols for using those data (Bird et al. 2021)

PEOPLE 3000's goals in Phase 2 of the project are to integrate radiocarbon and paleoecological/ paleoenvironmental data as well as information on social institutions, to develop coevolutionary models on carrying capacity, social integration, and data from paleoclimate and paleoecology. To date we have collected data from 17 core case study regions and will work on issues of quality control and integrating data from each region.

PEOPLE 3000 is currently seeking members from around the world. We will hold open online meetings each May to present research findings from individual members and to recruit new case studies. Visit the PEOPLE 3000 website at pastglobalchanges. org/people3000 and sign up to the mailing list to be contacted by us on our upcoming meetings and activities.

\section{Upcoming activities}

We held a hybrid meeting to wrap up Phase 1 of the project in November 2021 (pastglobalchanges.org/calendar/27099), with separate working groups meeting in individual countries and communicating over Zoom. Our first online meeting of Phase 2 will take place in May 2022, open to all PEOPLE 3000 members and interested researchers.

\section{AFFILIATIONS}

Department of Anthropology and Institute of Archaeology, Baylor University, Waco, TX, USA 2Departamento de Ecología, Pontificia Universidad Católica de Chile, Santiago, Chile \& Institute of Ecology and Biodiversity, Santiago, Chile ${ }^{3}$ Anthropology Program and The Ecology Center, Utah State University, Logan, UT, USA

4Department of Anthropology and Center for Applied Archaeological Science, Boise State University, ID, USA

Millennium Nucleus UPWELL \& Center of Applied Ecology and Sustainability (CAPES), Santiago, Chile Department of Anthropology, Washington State University, Pullman, WA, USA

Max Planck Institute for the Science of Human History, Jena, Germany

\section{CONTACT}

Julie Hoggarth: Julie_Hoggarth@baylor.edu Claudio Latorre: clatorre@bio.puc.c

\section{REFERENCES}

Odling-Smee FJ et al. (2003) Niche construction: The neglected process in evolution. Princeton University Press, $488 \mathrm{pp}$

Freeman J et al. (2021) Hum Ecol 49: 369-382 Bird D et al. (2021) tDAR: doi:10.48512/XCV8459164 